\title{
Regional Imbalance in the Levels of Development in Bagalkot District- Karnataka: A Spatial Analysis
}

\author{
${ }^{1}$ Dr. L.T. Nayak and ${ }^{2}$ Mr. B.D. Ajjodi \\ ${ }^{1}$ Associate Professor and Research Guide, Karnatak Science College, Dharwad \\ ${ }^{2}$ Research Scholar, Karnatak University, Dharwad
}

\begin{abstract}
After independence India has made remarkable progress in the socio-economic status of the people. But the attainment of this has spread highly uneven among the urban and rural areas. Regional imbalances are common phenomenon in the world, which occurred across different continents, countries and regions in different extent and India is not exception in this context. This phenomenon spread among inter-regions, interstates and intra-states $n$ India. This resulted into uneven development in the country. This process of imbalance is not exception in Bagalkot district. There are three major rivers namely the Krishna, the Malaprabha and the Ghataprabha and their tributaries flow in the study area and play important role in the development of the district. However, the study reveals that there exists a wide imbalance in the level of socio-economic development in the region. In view of this, an attempt has been made in this research work to evaluate and compares temporal growth and spatial pattern of disparities in the levels of socio-economic development at block level in Bagalkot district. Correlation coefficient technique has been applied to understand the correlation between different factors which are responsible for widening the regional imbalance. To analyses the regional imbalance of six taluks of Bagalk.ot district, 23 social, economic and demographic indicators have been considered. The present paper is based on secondary information based on which the socio-economic imbalance among the talukes of the district is made with the help of composite development index for two time of point i.e. 1997 and 2017 data. It has been observed that the social indicator values varied from 11 to 27. The study period witnessed Bagalkot, Badami, Hunagund and Jamkhandi are the three taluks which represent high social development, whereas low $(<11)$ social development was observed in Bilagi taluk. The outstanding causes for this backwardness are are, less number of education institutes, communication centers, adult education centers, bealth centers, family planning centers, recreational centers etc. The observed values of economic development are ranging between 19 and 35. It had been observed in only two taluks viz Bilagi and Hungund were found in high economic development, and low economic development was recorded in Jamakhandi taluk. Bagalkot, Mudhol and Badami taluks registered under relatively developed range. Lastly to understand the overall development, the observed values of socio-economic, economic and infrastructure indicators are added and derived a separate composite index.
\end{abstract}

Keywords: Regional imbalance, Socio-economic Development, Indicators, Demographic, Overall, Composite

\section{Article Publication}

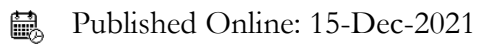

*Author's Correspondence

8 Dr. L.T. Nayak

8 Associate Professor and Research Guide, Karnatak Science College, Dharwad

$\triangle$ nayaklt68[at]gmail.com

C 2021The Authors. Published by RESEARCH REVIEW International Journal of Multidisciplinary. This is an open access article under the CC BY-

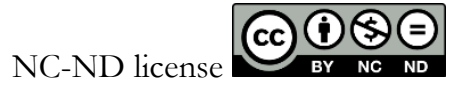

(https://creativecommons.org/licenses/ by-nc-nd/4.0/)

\section{Introduction}

The earlier research works reveal that the regional disparities are unique and universal phenomena in the world, which spread among the different countries and regions in different scale and economies. The major reasons, nature of occurrence and behavior of such disparities drew the attention of Geographers, regional planner, policy makers' researchers and economists. Scholars from different disciplines opined that the suitable method to study the levels of economic development is to analyze the economic growth of a country or region. There were several theories and models have been formulated by different scholars to answer this issue but the course remained 
unsolved. Myrdal (1957) and Kaldor (1970) argued that "basic forces at work are dis-equilibrating in nature. They tried to put forth the argument that once the divergence from equity occurs, the forces at work would be such that there is further divergence". Hirschman (1959) points out that the "polarization effects are stronger than the trickle-down effects in earlier stages of development of a nation. These and other such arguments brought on the scene the U-shaped curve between the extent of regional disparity and the level of national development". This has been empirically tested and proved by Kuznets (1958) and Williamson (1965). Regional Development includes the reorganization and reorientation of the entire economic and social system of a region or country, and balanced human resource development is one of the responsible factors for it. Government of India and Karnataka has implemented several developmental programmes to improve the quality and social and economic wellbeing of the people by providing the basic necessities. Backward regions are to be developed by implementing new government programmes like subsidies and Central assistance Socio-economic development is a process of betterment for a large human group and includes both economic development and social transformation. This improves the social condition of the people of the region.

The "report of the Inter-Ministerial Working Group on Redressing Growing Regional Imbalances (Planning Commission 2005) identified 170 most backward districts including 55 Extremist affected districts in the country. It has come out with suggestions which have tried to touch upon issues relating to decentralized governance with a focus on development of systematic village level plans".

Panchamukhi, (1998), Vyasalu, (1995), Vidwans (1996), Nanajundappa (1999), Abdul Aziz (2001), Hanagodimath (2006), Vivekananda (1992), Gayithri (1999), Nayak L.T (2004) and others have opined that recent past "regional imbalances in India is mostly a subject for the intra-state analysis than the inter-state". In this way there are many scholars worked on regional imbalances at the state level, but comparatively very a smaller number of studies carried out at the district level of an individual state. The study on regional imbalances within a state is more important than that of inter-state. Government of India has taken the initiation to set up various working committees for the redressal of regional imbalances in all states. In Karnataka regional imbalances have always been seen as dividing the state into three parts namely, Old Mysore, Hyderabad Karnataka and Bombay Karnataka.

As the (Planning commission of India) "Eleventh Plan commences, that a widespread perception all over the country is that disparities among States, and regions within States, between urban and rural areas, and between various sections of the community, have been steadily increasing in the past few years and that the gains of the rapid growth witnessed in this period have not reached all parts of the country and all sections of the people in an equitable manner". Though there is some evidence to indicate a movement towards convergence on human development indicators across States, one of the reasons for this convergence could also be that most human development indicators have a value cap. However, widening income differentials between more developed and relatively poorer States is a matter of serious concern. "The objective of the Eleventh Plan is 'faster and more inclusive growth' and each chapter has dealt with what the Plan proposes to do to achieve this objective, insofar as the sector dealt with by that chapter is concerned" (Planning commission of India).

\section{Study Region}

The study area Bagalkot district was a part of undivided Dharwad district till 1997, With re-organization of the district in 1997, the new Baglakot district come into exist during 50 th year of the India's independence. The study area consists of six blocks such as Badami, Bagalakot, Bilagi, Hunagunda, Jamakandi and Mudhol. It situated in the extreme northern part of Karanatak state lies between $16^{\circ} 07^{I} \mathrm{~N} 16^{\circ} 12^{\mathrm{I}}$ North latitude and $73^{\circ} 27^{\mathrm{I}}$ East $75^{\circ}$

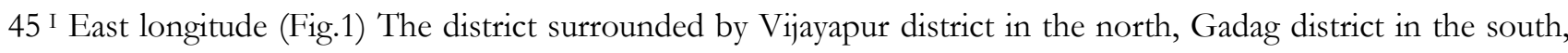
Raichur district towards the east, Koppal district to south east and Belgum district towards the west. It extends over an area of 6,593 sq kms with a population of 18, 89,752 persons (2011). Out of the total population 12,91,906 (70.2 per cent) are residing in rural areas and remaining 5,97,846 (29 percent) in Urban areas. The area under study accounts for 2.5 and 3.6 per cent of the state area and population respectively. Krishna, 
Ghataprabha, Malaprabha and their tributaries drain the district. District includes 566 villages, 15 towns and cities. Since the study is confined to the rural areas; hence towns and cities of the district are excluded from the study region.

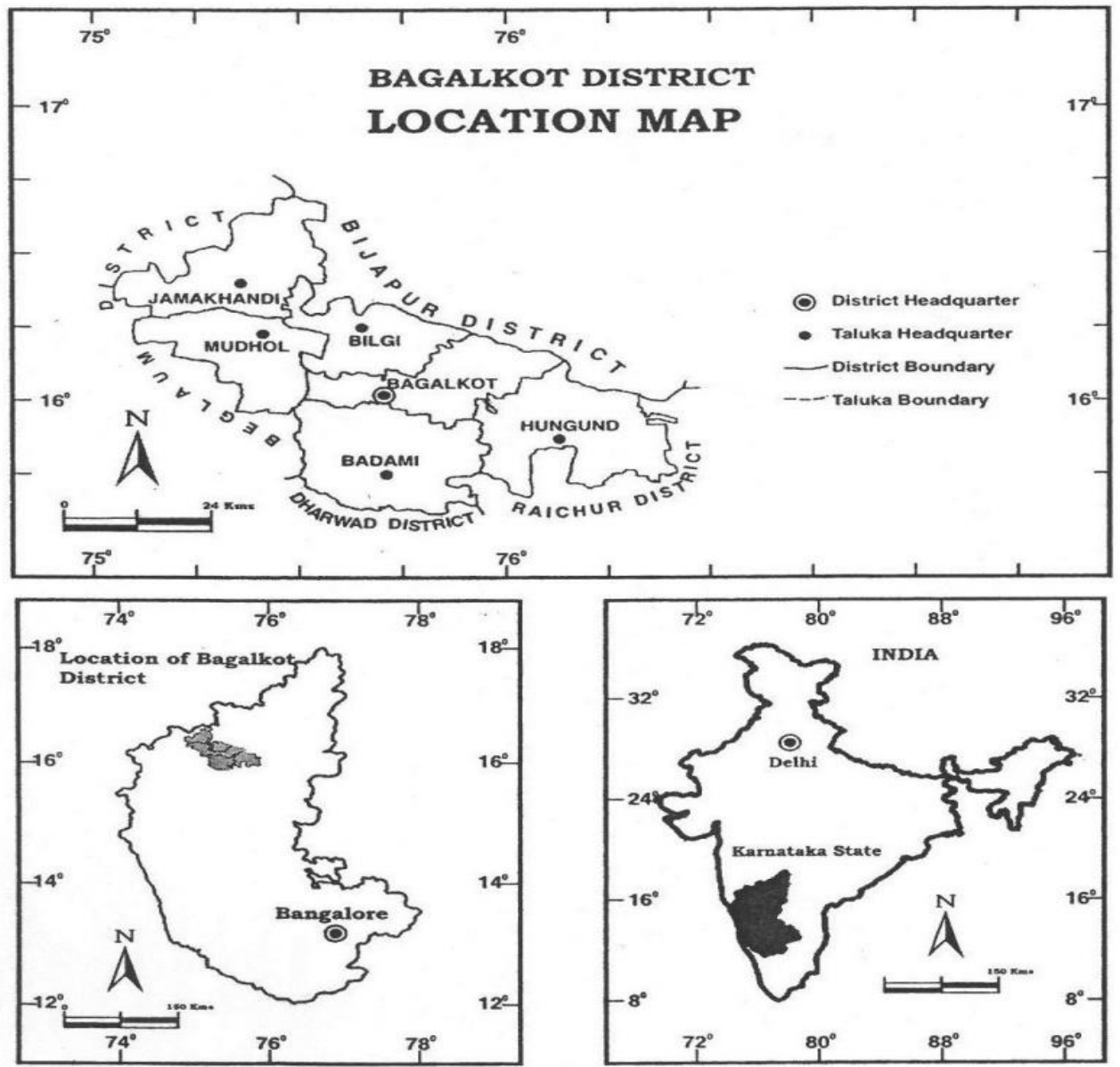

Source : National Atlas of India

Fid. 1

Location of Bagalkot district in Karnataka Location of Karnataka in India

Objectives:

The present research work is outlined with following objectives

1. To examine various levels of regional development considering the social, economic and demographic indicators.

2. To identify the socio-economically backward talukas and categories them into various levels of development in Bagalakot district.

3. To find out reasons responsible for regional imbalance in the study area.

4. To find out the correlation between demographic and social development and demographic and economic development

5. To find out the overall development level.

6. To suggests some measure to minimize the regional imbalance.

Hypotheses:

To achieve the above-mentioned objectives the following hypotheses have been formulated

- There exists a least positive correlation among different variables especially between demographic and social factors, and positive relation between demographic and combined development.

- The growth of agricultural production is not keeping pace with the growth of population. 
Database and Methodology:

A strong data base is required for qualitative research work and the present study is not exception of it. This research work is carried out with the help of secondary data collected from the district, at a glance 1997, Census hand books, Directorate of health and Family Welfare Service., Karnataka Commissioner for Public Instruction, Karnataka census of India (2011). A comprehensive composite index has been driven from a set of variables of a wide verity of multivariate statistical techniques. Considering the type of problem, the nature of the data and objective of the analysis, composite index method has been applied in the present study. The composite development index at taluk level of Bagalkot district has been derived and a composite picture has obtained through the following formula.

$$
\begin{aligned}
& \mathrm{CI}=\frac{\text { Observed value }}{\text { Mean of the respective column }} \\
& \mathrm{CI}=\text { composite index }
\end{aligned}
$$

The prime objective of the method for computing the composite index was to preservation of real variations among the different observations. Keeping this in view, the method of division by mean of the respective column (in the present analysis) has been preferred as it can get rid of the extreme values in the series. As far as the "determination of weights is concerned, no special weights have been assigned to any variable. (In other words, equal weightage has been assigned to all the selected variables to compute the development of region). After the removal of the biasness of scale, transformed values of the given variables are added up linearly and the scored values of composite index are classified into different categories" (Nayak L.T 2010). Categories such as high, medium and low developed regions have been made with the help of standard deviation grouping technique for combined score of 27 indicators.

\section{Selection of the variables:}

A large number of factors are available for identifying the regional disparities. But, based on the availability of data 27 indicators have been selected to examine the regional disparities in the levels of development of Bagalakot district and these indicators are broadly grouped into social, economic, and demographic indicators. They are mentioned below.

Table 1: Variables Groups, Description, Mean and Standard deviation-1997 \& 2017

\begin{tabular}{|c|c|c|c|c|}
\hline \multicolumn{1}{|c|}{ Social Indicators } & \multicolumn{3}{c|}{ Description } \\
\hline \multirow{2}{*}{ Sroups Indicator } & \multicolumn{2}{|c|}{1997} & \multicolumn{2}{c|}{2017} \\
\cline { 2 - 6 } X1 Primary Schools: Primary Schools per 10,000 population & Mean & SD & Mean & SD \\
\hline X2 High Schools: High Schools per 10,000 population & 9.27 & 18.99 & 10.07 & 8.76 \\
\hline X3 Colleges: PU and Degree colleges per 10,000 population & 1.51 & 0.56 & 2.45 & 0.07 \\
\hline X4 Post Office: Post and Telegraph office per 10,000 population & 0.48 & 0.08 & 0.73 & 0.30 \\
\hline X5 Hospitals: Hospitals per 10,000 population & 2.44 & 0.61 & 1.81 & 0.20 \\
\hline X 6 Dispensaries: Dispensaries per 10,000 population & 0.08 & 0.17 & 0.19 & 0.47 \\
\hline X7 $\begin{array}{l}\text { Primary Health care Unit: Primary Health Care centers per 10,000 } \\
\text { population }\end{array}$ & 0.12 & 0.10 & 0.45 & 0.17 \\
\hline X8 Cinema talkies: Cinema Talkies per 10,000 population & 0.03 & 0.09 & 0.24 & 0.08 \\
\hline X9 Family Planning Centers Family: Planning Centers per 10,000 & 0.14 & 0.08 & 0.24 & 0.11 \\
\hline X10 Library: Library per 10,000 population & 1.65 & 0.21 & 0.17 & 0.04 \\
\hline
\end{tabular}




\begin{tabular}{|l|c|c|c|c|}
\hline \multicolumn{1}{|c|}{ Groups Indicator } & \multicolumn{3}{c|}{ Description } \\
\hline \multicolumn{1}{|c|}{ II-Economic Indicators } & \multicolumn{2}{|c|}{1997} & \multicolumn{2}{c|}{2017} \\
\cline { 2 - 5 } & Mean & SD & Mean & SD \\
\hline X1. Registered Industries: No. Rgd Industries per 10,000 population & 4.35 & 1.27 & 56.36 & 14.34 \\
\hline X2. Commercial Banks: No of CB per 10,000 population & 0.54 & 0.17 & 0.71 & 0.35 \\
\hline $\begin{array}{l}\text { X3. Credit Societies: No. of Agriculture credit societies per 10,000 } \\
\text { population }\end{array}$ & 0.24 & 0.10 & 3.30 & 0.23 \\
\hline $\begin{array}{l}\text { X4. Co-operative society: No. of co-operative society per 10,000 } \\
\text { population }\end{array}$ & 0.52 & 0.14 & 0.70 & 0.50 \\
\hline X5 Telephones: Telephones in use per 10,000 population & 0.58 & 0.13 & 64.26 & 40.04 \\
\hline X6 Roads: Length of roads per 100sq kms of geographical area & 7.89 & 2.93 & 34.82 & 25.88 \\
\hline X7 Net sown area: Net area sown per 100sq kms of geographical area & 66.67 & 10.11 & 80.79 & 9.32 \\
\hline X8 Net Irrigated area: Net irrigated area per 100sq kms of net sown area & 43.65 & 24.00 & 57.83 & 20.40 \\
\hline $\begin{array}{l}\text { X1 Fertilizers: Chemical fertilizers (in metric tons) per 100sq kms of net } \\
\text { sown area }\end{array}$ & 4.55 & 2.63 & 162.88 & 101.20 \\
\hline $\begin{array}{l}\text { X10 Area sown more than once: area under sown more than once 100sq } \\
\text { kms net sown area }\end{array}$ & 17.69 & 7.02 & 30.87 & 12.44 \\
\hline X11 Length of railway per 100sq kms of Geographical area & 0.52 & 0.95 & 3.49 & 0.87 \\
\hline
\end{tabular}

\begin{tabular}{|c|c|c|c|c|}
\hline Groups Indicator & \multicolumn{4}{|c|}{ Description } \\
\hline \multirow{2}{*}{ III-Demographic Indicators } & \multicolumn{2}{|c|}{1997} & \multicolumn{2}{|c|}{2017} \\
\hline & Mean & SD & Mean & SD \\
\hline X1 Density Population > Density pop (Crude) & 26.19 & 216.16 & 44.91 & 2.45 \\
\hline X2 Population Growth $>$ Growth pop in percent & 21.00 & 7.46 & 19.03 & 3.61 \\
\hline X3 Sex ratio: No. of Females per 1000 males & 984 & 14.27 & 990 & 2.91 \\
\hline X4 Female Literates $>\%$ Female literates to female population & 37.90 & 3.27 & 43.55 & 4.15 \\
\hline X5 Male literates $>\%$ Male literates & 68.90 & 6.79 & 70.46 & 3.83 \\
\hline $\begin{array}{l}\text { X6 Main working Population \% of main working Population } \\
\text { to Total Population }\end{array}$ & 0.42 & 0.27 & 0.87 & 0.02 \\
\hline
\end{tabular}

Table.2: Values of Economic indicators and Composite score-1997 \&2017

\begin{tabular}{|c|c|c|c|c|c|c|c|c|c|c|c|c|}
\hline \multirow{2}{*}{$\begin{array}{c}\text { Name of the } \\
\text { taluks }\end{array}$} & \multicolumn{12}{|c|}{ Economic Development Indicators- 1997} \\
\hline & $\mathrm{X} 1$ & $\mathrm{X} 2$ & $\mathrm{X} 3$ & $\mathrm{X} 4$ & $\mathrm{X} 5$ & $\mathrm{X} 6$ & $\mathrm{X} 7$ & $\mathrm{X} 8$ & $\mathrm{X} 9$ & $\mathrm{X} 10$ & $\mathrm{X} 11$ & $\mathrm{CI}$ \\
\hline Badami & $\begin{array}{l}3.19 \\
(0.73)\end{array}$ & $\begin{array}{l}0.5 \\
(0.92)\end{array}$ & $\begin{array}{l}0.3 \\
(1.29)\end{array}$ & $\begin{array}{l}0.50 \\
(0.96)\end{array}$ & $\begin{array}{l}0.46 \\
(1.21)\end{array}$ & $\begin{array}{l}10.09 \\
(1.27)\end{array}$ & $\begin{array}{l}48.93 \\
(0.73)\end{array}$ & $\begin{array}{l}28.23 \\
7.73\end{array}$ & $\begin{array}{l}3.92 \\
(0.84)\end{array}$ & $\begin{array}{l}18.84 \\
(0.26)\end{array}$ & $\begin{array}{l}2.57 \\
(0.73)\end{array}$ & 16.67 \\
\hline Bagalakot & $\begin{array}{r}6.09 \\
1.4)\end{array}$ & $\begin{array}{l}0.64 \\
(1.18)\end{array}$ & $\begin{array}{l}0.14 \\
(0.58)\end{array}$ & $\begin{array}{l}0.59 \\
(1.13)\end{array}$ & $\begin{array}{l}0.49 \\
(0.84)\end{array}$ & $\begin{array}{l}8.86 \\
(1.12)\end{array}$ & $\begin{array}{l}71.21 \\
(1.06)\end{array}$ & $\begin{array}{l}21.98 \\
(0.50)\end{array}$ & $\begin{array}{l}6.08 \\
(1.33)\end{array}$ & $\begin{array}{l}20.7 \\
(1.17)\end{array}$ & $\begin{array}{l}4.48 \\
(1.27)\end{array}$ & 13.12 \\
\hline Bilagi & $\begin{array}{l}3.44 \\
(0.74)\end{array}$ & $\begin{array}{l}0.55 \\
(1.07)\end{array}$ & $\begin{array}{l}0.33 \\
(1.37)\end{array}$ & $\begin{array}{l}0.25 \\
(0.48)\end{array}$ & $\begin{array}{l}0.84 \\
(1.44)\end{array}$ & $\begin{array}{l}2.81 \\
(0.35)\end{array}$ & $\begin{array}{l}55.26 \\
(1.06)\end{array}$ & $\begin{array}{l}55.49 \\
(1.27)\end{array}$ & $\begin{array}{l}0.05 \\
(0.01)\end{array}$ & $\begin{array}{l}11.69 \\
(0.66)\end{array}$ & - & 8.5 \\
\hline Hunagunda & $\begin{array}{l}3.91 \\
(0.08) \\
\end{array}$ & \begin{tabular}{|l|}
0.61 \\
$(1.12)$ \\
\end{tabular} & $\begin{array}{l}0.23 \\
(0.95) \\
\end{array}$ & $\begin{array}{l}0.48 \\
(0.92) \\
\end{array}$ & $\begin{array}{l}0.52 \\
(0.89)\end{array}$ & $\begin{array}{l}11.52 \\
(1.46)\end{array}$ & $\begin{array}{l}75.45 \\
(1.13) \\
\end{array}$ & $\begin{array}{l}7.88 \\
(0.18) \\
\end{array}$ & $\begin{array}{l}2.68 \\
(0.58) \\
\end{array}$ & $\begin{array}{l}7.77 \\
(0.43) \\
\end{array}$ & - & 8.04 \\
\hline Jamakhandi & $\begin{array}{l}3.32 \\
(0.76)\end{array}$ & $\begin{array}{l}0.55 \\
(0.01)\end{array}$ & $\begin{array}{l}0.29 \\
(1.20)\end{array}$ & $\begin{array}{l}0.58 \\
(1.11)\end{array}$ & $\begin{array}{l}0.50 \\
(0.86)\end{array}$ & $\begin{array}{l}5.3 \\
(0.67)\end{array}$ & $\begin{array}{l}73.67 \\
(1.10)\end{array}$ & $\begin{array}{l}70.21 \\
(1.60)\end{array}$ & $\begin{array}{l}7.53 \\
(1.65)\end{array}$ & $\begin{array}{l}17.51 \\
(0.98)\end{array}$ & - & 9.94 \\
\hline Mudhol & $\begin{array}{l}6.16 \\
(1.41) \\
\end{array}$ & $\begin{array}{l}0.40 \\
(0.74)\end{array}$ & $\begin{array}{l}0.45 \\
(1.87) \\
\end{array}$ & $\begin{array}{l}0.72 \\
(1.38)\end{array}$ & $\begin{array}{l}0.67 \\
(1.15)\end{array}$ & $\begin{array}{l}8.19 \\
(1.03)\end{array}$ & $\begin{array}{l}73.45 \\
(1.10) \\
\end{array}$ & $\begin{array}{l}76.86 \\
(1.76) \\
\end{array}$ & $\begin{array}{l}7.07 \\
(1.55)\end{array}$ & $\begin{array}{l}30.11 \\
(1.70)\end{array}$ & - & 14.04 \\
\hline Mean & 4.35 & 0.54 & 0.24 & 0.52 & 0.58 & 7.89 & 66.67 & 43.65 & 4.55 & 17.69 & 3.52 & \\
\hline
\end{tabular}




\begin{tabular}{|c|c|c|c|c|c|c|c|c|c|c|c|c|}
\hline \multirow{2}{*}{$\begin{array}{c}\text { Name of the } \\
\text { taluks }\end{array}$} & \multicolumn{12}{|c|}{ Economic Development Indicators- 2017} \\
\hline & $\mathrm{X} 1$ & $\mathrm{X} 2$ & $\mathrm{X} 3$ & $\mathrm{X} 4$ & $\mathrm{X} 5$ & $\mathrm{X} 6$ & $\mathrm{X7}$ & $\mathrm{X} 8$ & $\mathrm{X} 9$ & $\mathrm{X} 10$ & $\mathrm{X} 11$ & CI \\
\hline Badami & $\begin{array}{l}59.81 \\
(1.06)\end{array}$ & $\begin{array}{l}0.42 \\
(0.59)\end{array}$ & $\begin{array}{l}3.26 \\
(0.98)\end{array}$ & $\begin{array}{l}1.42 \\
(0.83)\end{array}$ & $\begin{array}{l}40.50 \\
(0.63)\end{array}$ & $\begin{array}{l}92.66 \\
(0.69)\end{array}$ & $\begin{array}{l}62.56 \\
(0.91)\end{array}$ & \begin{tabular}{|l|}
38.69 \\
$(0.66)$
\end{tabular} & $\begin{array}{l}83.16 \\
(0.51)\end{array}$ & $\begin{array}{l}41.82 \\
(1.35)\end{array}$ & $\begin{array}{l}2.61 \\
(0.74)\end{array}$ & 8.95 \\
\hline Bagalakot & $\begin{array}{l}68.14 \\
(1.20)\end{array}$ & $\begin{array}{l}1.12 \\
(1.57)\end{array}$ & $\begin{array}{l}3.19 \\
(0.96)\end{array}$ & $\begin{array}{l}1.60 \\
(0.97)\end{array}$ & $\begin{array}{l}49.62 \\
(0.77)\end{array}$ & $\begin{array}{l}111.65 \\
(0.83)\end{array}$ & $\begin{array}{l}71.20 \\
(1.03)\end{array}$ & \begin{tabular}{|l|}
36.25 \\
$(0.62)$
\end{tabular} & $\begin{array}{l}74.51 \\
(0.45)\end{array}$ & $\begin{array}{l}45.06 \\
(1.45)\end{array}$ & $\begin{array}{l}4.37 \\
(1.25)\end{array}$ & 11.1 \\
\hline Bilagi & $\begin{array}{l}61.13 \\
(1.08)\end{array}$ & $\begin{array}{l}0.68 \\
(0.95)\end{array}$ & $\begin{array}{l}3.99 \\
(0.20)\end{array}$ & $\begin{array}{l}2.37 \\
(1.39)\end{array}$ & $\begin{array}{l}35.99 \\
(0.56)\end{array}$ & $\begin{array}{l}151.19 \\
(1.12)\end{array}$ & $\begin{array}{l}50.47 \\
(0.73)\end{array}$ & \begin{tabular}{|l|}
87.67 \\
$(1.51)$
\end{tabular} & $\begin{array}{l}188.96 \\
(1.16)\end{array}$ & $\begin{array}{l}23.61 \\
(0.76)\end{array}$ & -- & 9.46 \\
\hline Hunagunda & $\begin{array}{l}61.92 \\
(1.09)\end{array}$ & $\begin{array}{l}0.49 \\
(0.69)\end{array}$ & $\begin{array}{l}2.98 \\
(0.90)\end{array}$ & $\begin{array}{l}1.18 \\
(0.69)\end{array}$ & $\begin{array}{l}33.08 \\
(0.51)\end{array}$ & $\begin{array}{l}132.20 \\
(0.98)\end{array}$ & $\begin{array}{l}79.25 \\
(1.15)\end{array}$ & \begin{tabular}{|l|}
14.00 \\
$(0.24)$
\end{tabular} & $\begin{array}{l}50.27 \\
(0.30)\end{array}$ & $\begin{array}{l}52.35 \\
(1.69)\end{array}$ & - & 8.24 \\
\hline Jamakhandi & $\begin{array}{l}24.84 \\
(0.44)\end{array}$ & $\begin{array}{l}0.48 \\
(0.67)\end{array}$ & $\begin{array}{l}3.91 \\
(1.18)\end{array}$ & $\begin{array}{l}1.14 \\
(0.67)\end{array}$ & $\begin{array}{l}63.74 \\
(0.99)\end{array}$ & $\begin{array}{l}154.76 \\
(1.15)\end{array}$ & $\begin{array}{l}73.86 \\
(1.07)\end{array}$ & \begin{tabular}{|l|}
80.39 \\
$(1.39)$
\end{tabular} & $\begin{array}{l}273.52 \\
(0.14)\end{array}$ & $\begin{array}{l}10 . .0 \\
(0.32)\end{array}$ & - & 80.2 \\
\hline Mudhol & $\begin{array}{l}62.36 \\
(1.10)\end{array}$ & $\begin{array}{l}1.11 \\
(1.56)\end{array}$ & $\begin{array}{l}2.51 \\
(0.76)\end{array}$ & $\begin{array}{l}1.39 \\
(0.81)\end{array}$ & $\begin{array}{l}62.64 \\
(0.97)\end{array}$ & $\begin{array}{l}166.99 \\
(1.24)\end{array}$ & $\begin{array}{l}73.62 \\
(1.07)\end{array}$ & $\begin{array}{l}90.02 \\
(1.55)\end{array}$ & $\begin{array}{l}306.87 \\
(1.88)\end{array}$ & $\begin{array}{l}12.38 \\
(0.40)\end{array}$ & - & 11.34 \\
\hline Mean & 26.36 & 0.71 & 3.30 & 1.70 & 64.26 & 134.22 & 68.49 & 57.83 & 162.88 & 30.87 & 3.49 & \\
\hline
\end{tabular}

Regional variations in the levels of Economic Development

To examine the economic development of the district agriculture, industries, bank, societies, telephones, transports are the important indicators taken into consideration and composite index has been drawn and thereafter the obtained composite values have been presented in table 2. It has been observed from the fact that Badami (15.94) Bagalkot (11.85) and Mudhol (12.94) talukas performed higher economic development during 1997, It is mainly because of two economic sectors like agricultural and industries have been exceptionally developed. During 2017 Mudhol (11.34) and Bagalkot (9.85) talukas retained their first position of higher economic development where as Badami taluk shifted from high economic development to low economic development. During 1997 and 2017 low economic development was recorded in Hunagunda (8.04), Bilagi (8.5) and Jamkhandi (8.02) talukas except Badami taluk which was registered under low development in 2017 but its position was medium in 1997. This low economic development is due to the fact that economic indicators mainly commercial banks, agricultural, credit, societies, co-operative societies, length of the road, length of the railway lines, net area sown and net irrigated area are less in number and area and their development is also at low level. During 1997 Jamakhandi taluka is shown medium economic development, and during 2017 Bilagi and Bagalkot taluks fall under medium economic development category. It is pertinent to mention that the economic position of Bilgi taluk marginally improved during the study period. Earlier this was in low economic development range but because of improvement it shifted under medium economic development category. (Fig.2)

\section{Regional variations in the levels of Social Development}

Social indicators are important as they give clear idea about the disparities in any area under study. The social indicator includes schools and colleges, family planning and recreational facilities. The composite index/score of social indicators have been computed for all the 6 taluks with the help of composite development index and the obtained score has been presented in Table. 3. It was absorbed from the social indicator values which are varied from 5.91 in Jamkhandi taluk to 11,86 in Hunagunda taluk in 1997. Hunagunda (11.86) and Bilagi(11.32) taluks registered under high range of social development in 1997. The same taluks namely Bilagi(13.54) and Hunagunda(12.3) have maintained the same status of high economic development even in 2017. The values of social indicators among taluk vary ranging from 6.68 in Jamkhandi taluk to 13.54 in Badami taluk. Low (Range <.91) value of social development was observed in Jamakhandi, (6.68). Badami (9.75), Bagalkot (10.14) and Mudhol (10.38) talukas in 2017. The reason for the low development is less number of primary schools, high schools, colleges less number of post office, less number of primary health care center, family planning centers, and cinema talkies, etc these infrastructure have not met the actual requirements as the population of the taluks are more. During study period i.e. 1997 and 2017 the medium social development indicates in Bagalakot (9.98), Bilgi (11.38) and Hunagund (12.23) taluks respectively. 
Table.3: Values of Social indicators and Composite score-1997 \&2017

\begin{tabular}{|c|c|c|c|c|c|c|c|c|c|c|c|}
\hline Name of the & \multicolumn{11}{|c|}{ Social Development Indicators- 1997} \\
\hline & $\mathrm{X} 1$ & $\mathrm{X} 2$ & $\mathrm{X} 3$ & $\mathrm{X} 4$ & $\mathrm{X} 5$ & X6 & $\mathrm{X} 7$ & $\mathrm{X} 8$ & X9 & $\mathrm{X} 10$ & $\mathrm{CI}$ \\
\hline Badami & $\begin{array}{l}10.33 \\
(1.11)\end{array}$ & $\begin{array}{l}1.51 \\
(1.00)\end{array}$ & $\begin{array}{l}0.46 \\
(0.95)\end{array}$ & $\begin{array}{l}3.50 \\
(1.43)\end{array}$ & $\begin{array}{l}0.07 \\
(0.87)\end{array}$ & $\begin{array}{l}0.03 \\
(0.25)\end{array}$ & $\begin{array}{l}0.31 \\
(0.03)\end{array}$ & $\begin{array}{l}0.15 \\
(1.07)\end{array}$ & $\begin{array}{l}1.87 \\
(1.13)\end{array}$ & $\begin{array}{l}0.81 \\
(1.10)\end{array}$ & 8.94 \\
\hline Bagalakot & $\begin{array}{l}8.74 \\
(0.94)\end{array}$ & $\begin{array}{l}2.19 \\
(1.45)\end{array}$ & $\begin{array}{l}0.79 \\
(1.64)\end{array}$ & $\begin{array}{l}2.49 \\
(1.02)\end{array}$ & $\begin{array}{l}0.09 \\
(1.12)\end{array}$ & $\begin{array}{l}0.04 \\
(0.33)\end{array}$ & $\begin{array}{l}0.24 \\
(0.08)\end{array}$ & $\begin{array}{l}0.09 \\
(0.64)\end{array}$ & $\begin{array}{l}1.54 \\
(0.93)\end{array}$ & $\begin{array}{l}0.74 \\
(0.01)\end{array}$ & 9.88 \\
\hline Bilagi & $\begin{array}{l}7.89 \\
(0.85)\end{array}$ & $\begin{array}{l}1.0 \\
(0.10)\end{array}$ & $\begin{array}{l}0.42 \\
(0.87)\end{array}$ & $\begin{array}{l}2.77 \\
(1.13)\end{array}$ & $\begin{array}{l}0.08 \\
(0.06)\end{array}$ & $\begin{array}{l}2.75 \\
(0.08)\end{array}$ & $\begin{array}{l}0.33 \\
(1.1)\end{array}$ & $\begin{array}{l}0.25 \\
(1.78)\end{array}$ & $\begin{array}{l}2.18 \\
(1.32)\end{array}$ & $\begin{array}{l}1.00 \\
(1.36)\end{array}$ & 11.32 \\
\hline Hunagunda & $\begin{array}{l}11.29 \\
(1.21)\end{array}$ & $\begin{array}{l}1.75 \\
(1.15)\end{array}$ & $\begin{array}{l}0.57 \\
(1.18)\end{array}$ & $\begin{array}{l}2.11 \\
(0.86)\end{array}$ & $\begin{array}{l}0.12 \\
(1.5)\end{array}$ & $\begin{array}{l}0.08 \\
(0.66)\end{array}$ & $\begin{array}{l}0.48 \\
(1.6)\end{array}$ & $\begin{array}{l}0.24 \\
(1.74)\end{array}$ & $\begin{array}{l}1.99 \\
(1.20)\end{array}$ & $\begin{array}{l}0.58 \\
(0.79)\end{array}$ & 11.86 \\
\hline Jamakhandi & $\begin{array}{l}8.28 \\
(0.8)\end{array}$ & $\begin{array}{l}1.14 \\
(0.7)\end{array}$ & $\begin{array}{l}0.47 \\
(0.9)\end{array}$ & $\begin{array}{l}1.53 \\
(0.62\end{array}$ & $\begin{array}{l}0.05 \\
(0.06)\end{array}$ & $\begin{array}{l}0.08 \\
(0.66)\end{array}$ & $\begin{array}{l}0.17 \\
(0.56)\end{array}$ & 0.0 & $\begin{array}{l}1.20 \\
(0.72)\end{array}$ & $\begin{array}{l}0.50 \\
(0.68)\end{array}$ & 5.91 \\
\hline Mudhol & $\begin{array}{l}9.11 \\
(0.9)\end{array}$ & $\begin{array}{l}1.49 \\
(0.9)\end{array}$ & $\begin{array}{l}0.22 \\
(0.4)\end{array}$ & $\begin{array}{l}2.26 \\
(0.9)\end{array}$ & $\begin{array}{l}0.09 \\
(1.1)\end{array}$ & $\begin{array}{l}0.18 \\
(1.5)\end{array}$ & $\begin{array}{l}0.24 \\
(0.9)\end{array}$ & $\begin{array}{l}0.13 \\
(0.92)\end{array}$ & $\begin{array}{l}1.13 \\
(0.68)\end{array}$ & $\begin{array}{l}0.4 \\
(0.67)\end{array}$ & 9.1 \\
\hline Mean & 9.27 & 1.51 & 0.48 & 2.44 & 0.08 & 0.12 & 0.3 & 0.14 & 1.65 & 0.73 & \\
\hline
\end{tabular}

\begin{tabular}{|c|c|c|c|c|c|c|c|c|c|c|c|}
\hline \multirow{2}{*}{$\begin{array}{c}\text { Name of the } \\
\text { taluks }\end{array}$} & \multicolumn{11}{|c|}{ Social Development Indicators- 2017} \\
\hline & $\mathrm{X} 1$ & $\mathrm{X} 2$ & $\mathrm{X} 3$ & $\mathrm{X} 4$ & $\mathrm{X} 5$ & $\mathrm{X} 6$ & $\mathrm{X} 7$ & $\mathrm{X} 8$ & $\mathrm{X} 9$ & $\mathrm{X} 10$ & $\mathrm{CI}$ \\
\hline Badami & $\begin{array}{l}9.33 \\
(0.92)\end{array}$ & $\begin{array}{l}2.26 \\
(0.92)\end{array}$ & $\begin{array}{l}1.29 \\
(1.76)\end{array}$ & $\begin{array}{l}2.14 \\
(1.18)\end{array}$ & $\begin{array}{l}0.03 \\
(0.15)\end{array}$ & $\begin{array}{l}0.48 \\
(1.06)\end{array}$ & $\begin{array}{l}0.27 \\
(1.12)\end{array}$ & $\begin{array}{l}0.21 \\
(0.87)\end{array}$ & $\begin{array}{l}0.12 \\
(0.70)\end{array}$ & $\begin{array}{l}7.13 \\
(1.07)\end{array}$ & 9.75 \\
\hline Bagalakot & $\begin{array}{l}9.0 \\
(0.89) \\
\end{array}$ & $\begin{array}{l}2.66 \\
(1.08) \\
\end{array}$ & $\begin{array}{l}1.22 \\
(1.67)\end{array}$ & $\begin{array}{l}1.64 \\
(0.90)\end{array}$ & $\begin{array}{l}0.03 \\
(0.15)\end{array}$ & $\begin{array}{l}0.45 \\
(1)\end{array}$ & $\begin{array}{l}0.21 \\
(0.81)\end{array}$ & $\begin{array}{l}0.24 \\
(1 .)\end{array}$ & $\begin{array}{l}0.21 \\
(1.23)\end{array}$ & $\begin{array}{l}8.97 \\
(1.35)\end{array}$ & 10.14 \\
\hline Bilagi & $\begin{array}{l}11.35 \\
(1.12)\end{array}$ & $\begin{array}{l}2.62 \\
(1.06) \\
\end{array}$ & $\begin{array}{l}0.81 \\
(1.10)\end{array}$ & $\begin{array}{l}1.99 \\
(1.09)\end{array}$ & $\begin{array}{l}0.62 \\
(3.26)\end{array}$ & $\begin{array}{l}0.81 \\
(1.8)\end{array}$ & $\begin{array}{l}0.12 \\
(0.5)\end{array}$ & $\begin{array}{l}0.12 \\
(0.5)\end{array}$ & $\begin{array}{l}0.24 \\
(1.41)\end{array}$ & $\begin{array}{l}11.35 \\
(1.70)\end{array}$ & 13.54 \\
\hline Hunagunda & $\begin{array}{l}10.17 \\
(1.00)\end{array}$ & $\begin{array}{l}2.52 \\
(1.02)\end{array}$ & $\begin{array}{l}0.74 \\
(1.01)\end{array}$ & $\begin{array}{l}2.24 \\
(1.23)\end{array}$ & $\begin{array}{l}0.46 \\
(2.42)\end{array}$ & $\begin{array}{l}0.34 \\
(0.75)\end{array}$ & $\begin{array}{l}0.40 \\
(1.66)\end{array}$ & $\begin{array}{l}0.28 \\
(1.16)\end{array}$ & $\begin{array}{l}0.15 \\
(0.88)\end{array}$ & $\begin{array}{l}7.31 \\
(1.10)\end{array}$ & 12.23 \\
\hline Jamakhandi & $\begin{array}{l}10.29 \\
(1.02)\end{array}$ & $\begin{array}{l}2.19 \\
(0.89)\end{array}$ & $\begin{array}{l}0.82 \\
(0.12)\end{array}$ & $\begin{array}{l}1.36 \\
(0.75)\end{array}$ & $\begin{array}{l}0.02 \\
(0.10)\end{array}$ & $\begin{array}{l}0.25 \\
(0.55)\end{array}$ & $\begin{array}{l}0.21 \\
(0.87)\end{array}$ & $\begin{array}{l}0.19 \\
(0.79)\end{array}$ & $\begin{array}{l}0.12 \\
(0.70)\end{array}$ & $\begin{array}{l}5.95 \\
(0.89)\end{array}$ & 6.68 \\
\hline Mudhol & $\begin{array}{l}30.31 \\
(3.00)\end{array}$ & $\begin{array}{l}2.48 \\
(1.01)\end{array}$ & $\begin{array}{l}0.77 \\
(1.05)\end{array}$ & $\begin{array}{l}1.49 \\
(0.82)\end{array}$ & $\begin{array}{l}0.03 \\
(0.15)\end{array}$ & $\begin{array}{l}0.37 \\
(0.82)\end{array}$ & $\begin{array}{l}0.27 \\
(1.12)\end{array}$ & $\begin{array}{l}0.31 \\
(0.29)\end{array}$ & $\begin{array}{l}0.18 \\
(1.05)\end{array}$ & $\begin{array}{l}7.17 \\
(1.07)\end{array}$ & 10.38 \\
\hline Mean & 10.07 & 2.45 & 0.73 & 1.81 & 0.19 & 0.45 & 0.24 & 0.24 & 0.17 & 6.64 & \\
\hline
\end{tabular}

Regional variations in the levels of Demographic Development

Density and growth of populations, sex ratio, male and female literacy, and working population are the important demographic indicators considered and composite values are found by applying composite index formula and shown in Table 4. It was observed from table. 4 that the demographic indicators values varied from 5.6 in Bilagi to 6.54 Mudhol in 1997. Jamakhandi, (6.16) Bagalkot, (6.3) Mudhol, talukas indicate high demographic development with a value of more than 5.57 where as low demographic development exhibits in the all remaining talukas. less population density, less population potentials, less percentage of total literates, less percentage of female literates, less percentage of main working population are the sticking reasons for low development. The demographic indicators result in 2017 were varying with ranging from 5.57 in Badami to 6.36 in Jamkhandi taluk. The high demographic development represents in only one taluk namely Jamkhandi (6.36). Mudhol (6.03) and Bagalkot (6.16) taluk come under medium range of demographic development. Low range of demographic values are found in Badami (5.50), Bilgi (5.62) and Hungund (5.78) taluks. 
Table.3: Values of Demographic indicators and Composite score-1997 \& 2017

\begin{tabular}{|l|l|l|l|l|l|l|l|}
\hline $\begin{array}{c}\text { Name of the } \\
\text { taluks }\end{array}$ & \multicolumn{7}{|c|}{ Demographic Development Indicators- 1997 } \\
\hline \multirow{2}{*}{ Badami } & \multicolumn{1}{|c|}{ X1 } & \multicolumn{1}{|c|}{ X2 } & \multicolumn{1}{|c|}{ X3 } & \multicolumn{1}{|c|}{ X4 } & \multicolumn{1}{|c|}{ X5 } & \multicolumn{1}{|c|}{ X6 } \\
\hline \multirow{2}{*}{ Bagalakot } & 223 & 19.50 & 995 & 75.09 & 42.55 & 0.45 & 5.22 \\
& $(1.03)$ & $(0.69)$ & $(0.98)$ & $(1.10)$ & $(1.16)$ & $(0.95)$ & 5.91 \\
\hline \multirow{2}{*}{ Bilagi } & 152 & 21.12 & 1000 & 64.09 & 31.31 & 0.48 & 5.6 \\
& $(0.70)$ & $(1.00)$ & $(1.01)$ & $(0.93)$ & $(0.82)$ & $(1.14)$ & \\
\hline \multirow{2}{*}{ Hunagunda } & 181 & 11.40 & 999 & 75.41 & 39.8 & 0.43 & 5.52 \\
& $(0.83)$ & $(0.54)$ & $(1.01)$ & $(1.09)$ & $(1.03)$ & $(1.02)$ & \\
\hline \multirow{2}{*}{ Jamakhandi } & 290 & 25.24 & 964 & 60.51 & 34.04 & 0.38 & \multirow{2}{*}{5.84} \\
& $(1.01)$ & $(1.20)$ & $(0.97)$ & $(0.87)$ & $(0.89)$ & $(0.90)$ & \\
\hline \multirow{2}{*}{ Mudhol } & 231 & 34.21 & 978 & 62.06 & 36.04 & 0.43 & 6.54 \\
& $(1.06)$ & $(1.62)$ & $(0.99)$ & $(0.90)$ & $(0.95)$ & $(1.02)$ & \\
\hline Mean & 216 & 21.00 & 984 & 68.90 & 37.90 & 0.42 & \\
\hline
\end{tabular}

\begin{tabular}{|c|c|c|c|c|c|c|c|}
\hline \multirow{2}{*}{$\begin{array}{c}\text { Name of the } \\
\text { taluks }\end{array}$} & \multicolumn{7}{|c|}{ Demographic Development Indicators- 2017} \\
\hline & $\mathrm{X} 1$ & $\mathrm{X} 2$ & X3 & $\mathrm{X} 4$ & $\mathrm{X} 5$ & $\mathrm{X} 6$ & $\mathrm{CI}$ \\
\hline Badami & $\begin{array}{l}212 \\
(0.86)\end{array}$ & $\begin{array}{l}13.8 \\
(0.71)\end{array}$ & $\begin{array}{l}984 \\
(0.99)\end{array}$ & $\begin{array}{l}71.70 \\
(1.05)\end{array}$ & $\begin{array}{l}41.10 \\
0.94\end{array}$ & $\begin{array}{l}0.36 \\
(1.02)\end{array}$ & 5.57 \\
\hline Bagalakot & $\begin{array}{l}264 \\
(1.07)\end{array}$ & $\begin{array}{l}18.02 \\
(0.94)\end{array}$ & $\begin{array}{l}991 \\
(1.00)\end{array}$ & $\begin{array}{l}77.50 \\
(1.09)\end{array}$ & $\begin{array}{l}49.17 \\
(1.12)\end{array}$ & $\begin{array}{l}0.33 \\
(0.94)\end{array}$ & 6.16 \\
\hline Bilagi & $\begin{array}{l}183 \\
(0.74)\end{array}$ & $\begin{array}{l}19.3 \\
(1.01)\end{array}$ & $\begin{array}{l}1000 \\
(1.01)\end{array}$ & $\begin{array}{l}65.00 \\
(0.92)\end{array}$ & $\begin{array}{l}36.80 \\
(0.87)\end{array}$ & $\begin{array}{l}0.37 \\
(1.07)\end{array}$ & 5.62 \\
\hline Hunagunda & $\begin{array}{l}211 \\
(0.86)\end{array}$ & $\begin{array}{l}17.0 \\
(0.89)\end{array}$ & $\begin{array}{l}987 \\
(0.99)\end{array}$ & $\begin{array}{l}76.10 \\
(1.08)\end{array}$ & $\begin{array}{l}43.20 \\
(0.99)\end{array}$ & $\begin{array}{l}0.34 \\
(0.97)\end{array}$ & 5.78 \\
\hline Jamakhandi & $\begin{array}{l}350 \\
(1.42)\end{array}$ & $\begin{array}{l}0.4 \\
(1.07)\end{array}$ & $\begin{array}{l}983 \\
(0.99)\end{array}$ & $\begin{array}{l}67.70 \\
(0.96)\end{array}$ & $\begin{array}{l}40.30 \\
(0.92)\end{array}$ & $\begin{array}{l}0.35 \\
(1)\end{array}$ & 6.36 \\
\hline Mudhol & $\begin{array}{l}250 \\
(1.02)\end{array}$ & $\begin{array}{l}25.6 \\
(1.34)\end{array}$ & $\begin{array}{l}998 \\
(1.00)\end{array}$ & $\begin{array}{l}64.80 \\
(0.91)\end{array}$ & $\begin{array}{l}40.20 \\
(0.92)\end{array}$ & $\begin{array}{l}0.39 \\
(1.11)\end{array}$ & 5.3 \\
\hline Mean & 245 & 19.03 & 990 & 70.46 & 43.53 & 0.35 & \\
\hline
\end{tabular}

Table-4: Categories of Spatio- Temporal Development of Social, Economic and Demographic- 1997-2017

\begin{tabular}{|c|c|c|c|c|c|c|}
\hline \multicolumn{7}{|c|}{1997} \\
\hline Status & Range & Social & Range & Economic & Range & Demography \\
\hline Low & $>9.50$ & $\begin{array}{l}\text { Badami,Jamkhandi } \\
\text { \& Mudhol }\end{array}$ & $>11.52$ & $\begin{array}{l}\text { Hunagunda } \\
\text { Jamkahndi \& } \\
\text { Bilagi, }\end{array}$ & Below 5.77 & $\begin{array}{l}\text { Bagalakot,Badami } \\
\text { \&Hunagunda }\end{array}$ \\
\hline Medium & 9.50 to 11.42 & $\begin{array}{l}\text { Bagalakot, Bilagi } \\
\&\end{array}$ & 11.50 to 16.09 & $\begin{array}{l}\text { Mudhol, } \\
\text { Bagalakot }\end{array}$ & 5.77 to 6.17 & $\begin{array}{l}\text { Jamkhandi\& } \\
\text { Bagalakot }\end{array}$ \\
\hline High & Above 11.42 & Hunagunda & Above 16.09 & Badami & Above 6.17 & Mudhol \\
\hline
\end{tabular}




\begin{tabular}{|c|c|c|c|c|c|c|}
\hline \multicolumn{7}{|c|}{2017} \\
\hline Status & Range & Social & Range & Economic & Range & Demography \\
\hline Low & $>10.45$ & $\begin{array}{l}\text { Jmakhandi,Badami,B } \\
\text { agalakot \& Muhol }\end{array}$ & 9.51 to 10.8 & $\begin{array}{l}\text { Jamakhndi, } \\
\text { Hunagunda, } \\
\text { Badami\& } \\
\text { Bilagi }\end{array}$ & Below 5.91 & $\begin{array}{l}\text { Badami,Bilagi\& } \\
\text { Hunagunda }\end{array}$ \\
\hline $\begin{array}{l}\text { Mediu } \\
\mathrm{m}\end{array}$ & $\begin{array}{l}10.45 \\
\text { to } \\
12.61\end{array}$ & Hunagunda & 9.51 to 10.8 & Nil & 5.96.to 6.27 & $\begin{array}{l}\text { Mudhol,Bagala } \\
\text { kot }\end{array}$ \\
\hline High & $\begin{array}{l}\text { Above } \\
12.61\end{array}$ & Bilagi & Above 10.8 & $\begin{array}{l}\text { Bagalakot\& } \\
\text { Mudhol }\end{array}$ & Above 6.27 & Jamakhandi \\
\hline
\end{tabular}

Areas of high development

In this category three taluk of Bagalkot district viz, Badami (30.1) Mudhol (28.58) and Bilagi (28.62) showed high over all development (Table 5 \& 6). Based on three broad groups of indicators namely social, economic and demographic the development of these taluks has been examined. Badami, Mudhol and Bilagi taluk are well connected by the roads and railways (Fig.2). These taluks recorded first and second place in indicators like, colleges, number of registered factories, commercial banks, agricultural credit societies, co-operative societies, communication facility, agriculture implements, length of roads, length of railways lines, percentage of workers in economic activities have attracted the higher level of development of financial institutions and other economic and social infrastructure and tourist centers.

Table 5: Indices of Social, Economic and Demographic Development (1997-2017

Overall Development

\begin{tabular}{|c|c|c|c|c|c|c|c|c|}
\hline \multicolumn{4}{|c|}{1997} & & \multicolumn{3}{|c|}{2017} & \multirow[b]{2}{*}{ Overall } \\
\hline Taluks & SC-CI & E-CI & D-CI & Overall & SC-CI & E-CI & D-CI & \\
\hline Badami & 8.94 & 16.67 & 5.22 & 30.1 & 9.75 & 8.95 & 5.57 & 23.53 \\
\hline Bagalakot & 9.88 & 13.12 & 5.91 & 27.24 & 10.14 & 11.1 & 6.16 & 26.15 \\
\hline Bilagi & 11.32 & 8.5 & 5.6 & 25.42 & 13.54 & 9.46 & 5.62 & 28.62 \\
\hline Hunagunda & 11.86 & 8.04 & 5.52 & 25.42 & 12.23 & 8.24 & 5.78 & 26.25 \\
\hline Jamakhandi & 5.91 & 9.94 & 5.84 & 21.69 & 6.68 & 8.02 & 6.36 & 21.06 \\
\hline Mudhol & 9.1 & 12.94 & 6.54 & 28.58 & 10.38 & 11.34 & 6.3 & 28.02 \\
\hline
\end{tabular}

Table.6. Categories Overall Development Social, Economic \& Demography

\begin{tabular}{|c|c|c|c|c|}
\hline Status & Range & 1997 & Range & 2017 \\
\hline Low & $>25.69$ & $\begin{array}{c}\text { Jamkhandi(21.69) } \\
\text { Bilagi, (25.42) } \\
\text { Hunagunda(25.42) }\end{array}$ & $\begin{array}{c}\text { Badami (21.06) } \\
\text { Jmakhandi,(23.53) }\end{array}$ \\
\hline Medium & 25.70 to 29.1 & $\begin{array}{c}\text { Bagalakot (27.24) } \\
\text { Mudhol(28.58) }\end{array}$ & 25.70 to 28.38 & $\begin{array}{c}\text { Bagalakot (26.15) } \\
\text { Hunagunda(26.25) }\end{array}$ \\
\hline High & Above 29.2 & Badami (30.1) & Above 28.39 & $\begin{array}{c}\text { Mudhol (28.58) } \\
\text { Bilagi (28.62) }\end{array}$ \\
\hline
\end{tabular}



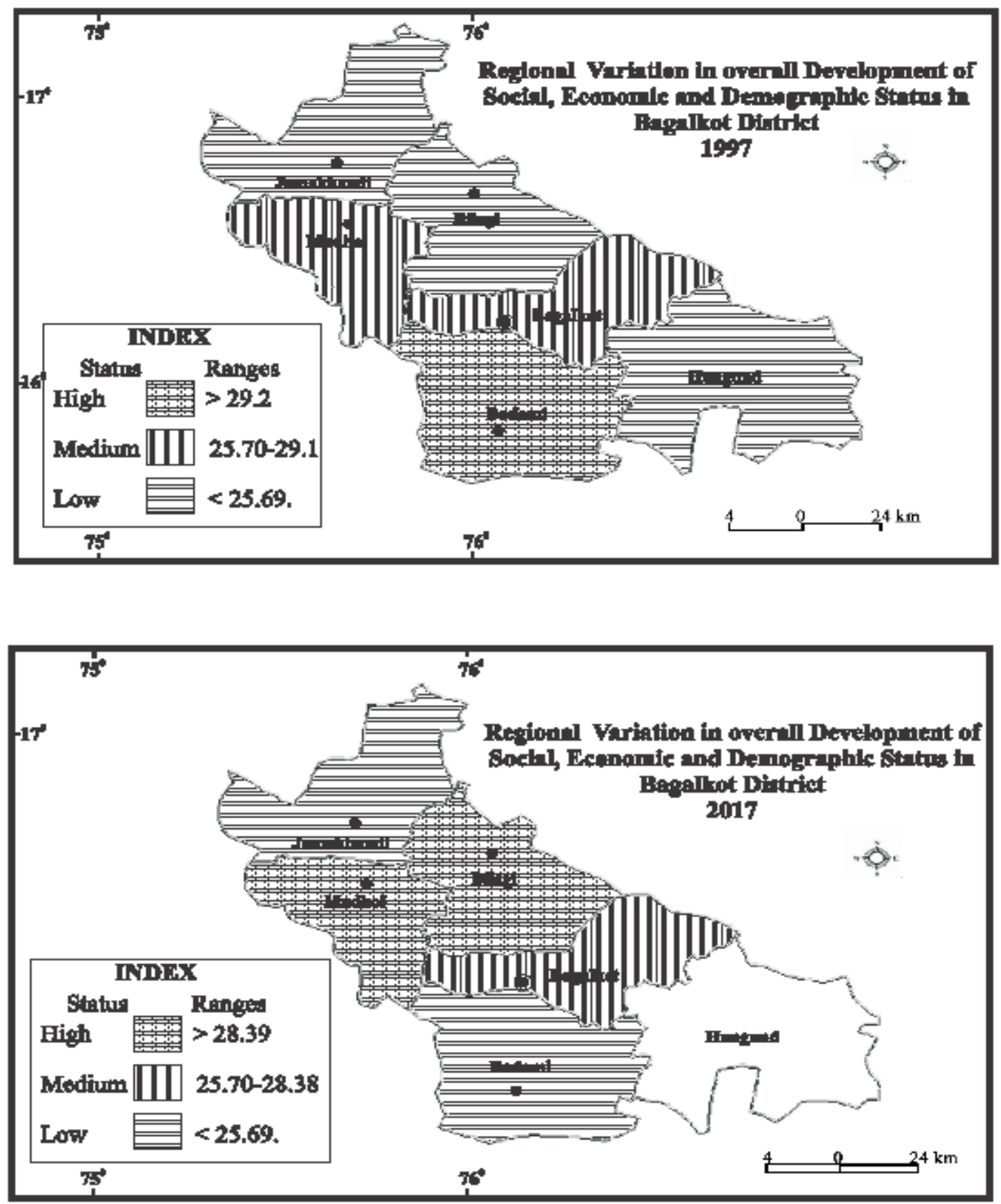

Fig.2

Areas of Medium developed

Mudhol (12.94) and Bagalakot (13.12) taluks falls under medium development category in 1997 where as in 2017 Bagalkot talik retain it position of medium development and Hungund taluk gain from low area of development to moderate development. 


\section{Areas of Low development}

During 1997 three taluks namely Jamkhandi(21.69) ,Hunagunda (25.42) and Bilagi(25.42) were less developed hence they fall under areas of low development with a range less than 29.69 value. In 2017 Badami taluk loses its earlier status of high development and fall into Low development, Jamkhandi taluk mentioned the same status of low development in 2017(Fig.3). Number of Industries are less, unfertile soil, less higher education facilities, poor communication and transport facilities, less irrigated area, less facility of credit societies and commercial banks are the striking reasons for the less development of these blocks. Badami, Hunagunda and Bagalakot taluks are endowed with natural resources particularly iron-ore and Dolomite minerals, but the extracted mineral ore and Granite stones are being exported to neighboring countries and profit gained the entrepreneurs. Disadvantage of topography and other natural resource are the other causes for the backwardness of these taluks. These taluks from the dry region of Karanatak with very low rainfall, poor type soil and so they are subjected to the frequent droughts and famine conditions.

The correlation coefficient among variable especially between social development with demographic and economic development, where demographic development on social as well as economic development have been found out as both are being independent in the present context. Karl's Pearsons's product moment co-relation coefficient technique has been implied. The co-relation between demographic development and social development is 0.12 being least positive correlation, while between demographic development and economic development is 0.84 positive correlations and between demographic and combined (social, Economic, and Demographic) development in 0.96 positive correlation ship. Therefore, in order to make further demographic development in Bellry district it is necessary to give priority to develop all the eight of the Bagalakot district.

\section{Conclusions}

The study region is located in semi-arid belt of mid Kaaranatak and which receives $575 \mathrm{~mm}$ annual rainfall in 39 rainy days spread over a period of six months. Balekundri left bank canal is constructed in the study area as a result of which 27.67 per cent of area under irrigated.

As the major rivers flow in the district which able to reduce the effects of drought and famine in some taluks viz., Jamakhandi, Badamiand Mudhol There were two taluks such as., Bagalakot, and Bilagi show high social development. Five taluks namely, Jamakhandi, Hunagunda, Bilagi, and Badami showed low social development.Two taluks viz., Bagalakot and Mudhol showed high economic development. Four taluks Jamakhandi, Hunagunda, Badami and Bilagi comes under medium economic development in 1997. There were three taluks viz., Bilagi, Hunagunda and Jamkhandi, which show low economic development. There were two taluks of Bagalakot district viz., Mudhol and Jamakhandi that indicate high demographic development. Four taluks of Bagalakot district viz., Bagaklakot, Badami, Hunagunda, and Jamakhandi showed overall development. This process has been found by assessing three broad functions, such as social, economic and demographic. The correlation between demographic development and social development is 0.12 being least positive correlation, while between demographic and economic development is 0.84 positive correlations and between demographic and combined (social, economic and demographic) development is positive correlations i.e., 0.96. 


\section{References}

Aziz Abdul (2001), Economic Development: Vision Strategies for the Future, in Jeevan Kumar and Susheela Subrahmanya (ed), Vision Karnataka 2025: Strategies and Action Plans for Sustanable Development, Southern Economist, Bangalore.

Gayithri (1999) Role of Infrastructure in Industrial Development of Karnataka: A district level analysis, , In Srinivas Gowda M.V . and Nanje Gowda (ed) 'Economic Evelopment of Karnataka: Leading Issues', the Local Advisory committee, 81 Annual Conference of the Indian Economic Association Bangalore.

Government of India, Planning Commission (2005): The report of the Inter-Ministerial Working Group on Redressing Growing Regional Imbalances.

Hanagodimath Shiddalingaswami (2006): Karnataka Arthic Belavanige Ondu Adyana - Yojana (Kannada), 2006.

Hirschman, A. O. (1959): The Strategy of Economic Development (New Haven: Yale University Press).

Kuznets, S. (1958): “Quantitative Aspects of Economic Growth of Nations-III: Industrial Distribution of Income and Labour Force by States, the United States, 1919-21 to 1955” in Economic Development and Cultural and Change, Vol. 6, July.

Mydral, G. (1957): Economic Theory and Underdeveloped Regions (London).

Nanjunhdappa D M (1999): Karnataka's Economy: Retrospect and Prospects, In Srinivas Gowda M.V. and Nanje Gowda (ed) 'Economic Evelopment of Karnataka: Leading Issues', the Local Advisory committee, 81 Annual Conference of the Indian Economic Association Bangalore.

Nayak, L.T and Narayankar, D.S. (2009): Identification of Regional Disparities in levels of development in Bellary

District-Karnataka, Indian Journal of Regional Science, 41(1): 37-47.

Panchamuchi P R (1998) :North, South Divide Karnataka's Development Scenario, Monograph No. 21, CMDR, Dharwad, Karnataka

Vidwas S M (1996): Regional disparity: A New Approach, Occasional Paper 1, Indian School of Political Economy, Pune. Vivekananda, M (1992): 'Agricultural Development and Distribution of Gains: An Intra and Inter Regional Analysis of Karnataka', Research Report, ISEC

Vyasalu Vinod (1995) : Management of Poverty Alleviation Programmes in Karnataka Economic and Political Weekly October 14-21

Williamson, J.G. (1965): “Regional Inequality and the Process of National Development: A Description of the Patterns" in Economic Development and Cultural Change, Vol. 13, No. 4, Part II, July. 